\title{
APPLICATION OF EYETRACKING RESEARCH TECHNIQUES INTO ROAD SAFETY STUDIES - THEORETICAL CONSIDERATIONS
}

\author{
Tomasz Kamiński, Mikołaj Kruszewski, Michał Niezgoda \\ Motor Transport Institute \\ Transport Telematics and Management Centre \\ Jagiellonska Street 80, 03-301 Warsaw, Poland \\ tel.: +48228113231, fax: +48228113231 \\ e-mail:tomasz.kaminski@its.waw.pl,mikolaj.kruszewski@its.waw.pl \\ michal.niezgoda@its.waw.pl
}

Pawel Soluch

Institute of Sensory Analysis

Plowiecka Street 1, 04-501 Warsaw, Poland

tel.: +4822 6621530, fax: +48224335007

e-mail:pawel@neurodevice.pl

\begin{abstract}
In recent years, both in Poland and throughout Europe the number of road accidents and the number of people injured and killed in these cases systematically decreases (despite a slight increase in 2011). Despite the general goals of EU transport policy for years 2000-2010 have not been fulfilled (the number of fatalities in road accidents have not been reduced by half in these years) the Commission on Mobility and Transport is continuing its efforts to reduce the number of accidents, which resulted in the EP resolution of 27th August, 2011. Under this policy, the EU Member States are forced to develop and implement appropriate procedures and requirements associated with road safety analysis in the near future. An important obligation have been introduced with the Directive 2008/96/EC, stating the compulsory road safety audits (RSA) should be carried on TEN-T road network. European Parliament also announced extension of this obligation on other public roads in the near future. These aspirations could be supported with modern engineering and research technology.

In following article, authors describe the possibilities of using eyetracking technologies in studies over road safety analysis. Therefore, modern eyetracking devices were characterized, with particular emphasis on its possible use in the study of driver's behaviours in road traffic conditions. Also, a brief analysis of the methodology of the eyetracking experiments in traffic conditions and their results was presented, indicating possibilities of carrying RSA using similar equipment. In the last chapter authors, discuss possible use of eyetracking technologies in studies of road safety analysis in the context of the existing law in European Union and each member's law regulation system.
\end{abstract}

Keywords: eyetracking, road safety audit (RSA), driver's behaviours, road safety analysis

\section{Introduction}

For over a decade, actions of the Directorate-General for Mobility and Transport have been aiming at significant reduction in the number of victims of road accidents and reduction of economic and social costs incurred by community. Improving road safety was the primary goal of the document published in 2001, called the White Paper "European transport policy for 2010, the time to decide", which is continued in the field of road safety in European Parliament resolution of 27 August 2011. The main objective of the current strategy is to reduce the number of road fatalities by $50 \%$ until year 2020 .

To implement this policy, the use of assessments and audits of road safety analysis (RSA) on public roads was provided. This obligation was introduced by European Parliament and Council 
Directive 2008/96/EC, which has been currently implemented in Poland. In its present form, the directive imposes obligatory evaluation of road safety and carrying road safety audits of all already existing and planned roads of the Trans-European Transport Network - TEN-T. This obligation in Poland should be fulfilled by the General Directorate for National Roads and Motorways (Polish GDDKiA - Generalna Dyrekcja Dróg Krajowych i Autostrad) and 27 presidents of cities with county rights. In their duties lies maintenance of a total number of about $4800 \mathrm{~km}$ of TEN-T road network in Poland. According to the Directive, road safety audits must be carried out either before the opening of newly constructed roads, as well as during their use, and additionally on roads with a high concentration of accidents. Directive also recommends the use of similar audits on other public roads.

Road safety auditor function is relatively new, in both Poland and other EU countries. This newly created market is currently based mainly on the knowledge and experience of auditors, while lack of solutions that could support their activities, mainly through automation and making evaluation more objective, and is highly noticeable. Due to the nature of the road traffic, best for this purpose may be eytracking tools - allowing the observation and analysis of the research subject's visual activity. These tools that have been already widespread for several decades in scope of scientific studies, recently reached a functionality allowing for wider dissemination - which can greatly expand the possibilities of their use in field of research in road safety analysis.

Following chapters briefly characterizes different types of eyetracking devices and their functional characteristics and limitations due to their application to road safety analysis studies. There have been also identified the current applications of eyetracking devices in studies related to road traffic, as well as characteristics and assumptions of carried experiments. There are also described sample analysis results.

\section{Main features of eye-trackers}

Eyetracking is a technique with more than 100-year history used in various scientific fields including issues, which require analysis of the human eye movement. Eyetracking study is performed by recording of the human visual activity during specific activities. First studies over human eye movement were carried out in a typically invasive way by placing the lens equipped with a special gap for pupil on subject's eye. Technological advances made it possible to change the way of gathering information about movement of the eye with the device mounted on the subject's head, making a completely non-invasive measurements by non-contact video observation of pupil movement.

We can distinguish two main divisions of these devices - by the way the unit is placed, and by the method of measurement. These are the following types of eyetracking devices (so called eyetrackers), according to the first division:

- static eyetracking devices, which is mounted on the table ahead the subject, and could appear as:

- mounted on a platform located in the close proximity of the subject, which reduces head movement. This design of the research stand results in a feeling of slight discomfort, but allows collecting data with high accuracy,

- remote, that does not restrict subject's head movement in any way and does not directly contact with him. It is a device with lower accuracy and lower quality of measurements, but providing far more subject's comfort (and in some cases is the only one possible solution to perform research e.g. newborns),

- eyetracking devices mounted on the head, where the lighting and recording equipment of the eye movements are mounted on a special helmet or headdress. Additional camera captures the real context scenes, which subject is actually looking at,

- eyetracking device mounted on the head with an additional equipment like i.e. head-tracker a device which records any movement in space of the head during the study.

For studies performed in road traffic the best solution are "remote" systems - i.e. installed in the vehicle. They do not affect freedom of movement and do not cause the driver's distraction, thus 
not affecting the natural process of driving. Mobile systems mounted on the subject's head also seem to be sufficient for this purpose. Modern systems of this type are installed in spectacle frames, which only slightly reduce the sense of freedom of the driver and is "unnatural" only for people that do not use glasses every day - for others level of distraction is still very low. In addition, worldwide studies show that during actions associated with high intellectual engagement (which includes also driving process), eyetracking devices do not change the subject's visual activity.

The use of static eyetracking devices is possible only if it is related with using visual material presented on the computer monitor such as videos and images, which due to their high accuracy are still a valuable option. But the lack of interaction with the vehicle has a major effect on the naturalness of the situation and provides that the tested person is more "passenger" rather than "driver".

Eyetrackers measure eye movements in several different ways, which sometimes differ from each other only in details. Using a slight generalization, eyetracking measurement methods can be divided into three categories:

- induction eyetracking - using an induction coil fixed directly to the eyeball,

- optical non-contact eyetracking,

- electro-eyetracking - the method of using electrodes placed around the eyes - which measure the electrical micropotentials between the cornea and retina.

Despite of the high efficiency achieved by using every of these methods, contemporary researches are conducted almost exclusively using non-contact optical method devices, as the least invasive and providing maximum comfort for the tested person. Technological progress, which particularly includes increasing of computational capabilities and miniaturization of computers made it possible to use very precise methods of an intelligent image analysis, achieving high-quality studies using optical methods involving the observation of eye movements by the video camera. Beam of infrared light is sent to the eye, which gets back to the device thanks to the reflection made on the surface of the eye and is detected by the optical sensor. Information is analyzed in terms of certain parameters related to its reflection, which allows determining the movements of the eye. Eyetrackers, which uses non-contact optical method, base on the detection of the centre of the eye pupil and the light reflection from cornea, so called first Purkinje image. Devices with higher sensitivity uses also the reflections forming in the back of the lens, and sometimes even track the location of blood vessels on the retina.

A single image of the eye captured by the camera and processes to determine the place which subject looks at is called a sample. The faster the system, the more samples are able to register in a second. The number of samples is directly relevant to the reliability of measurement results, so the frequency of the device must be adapted to the duration times of tested movements. For example, studying the microsaccade movements, which duration is between 10 and $30 \mathrm{~ms}$, the system should be using a sampling frequency of not less than $100 \mathrm{~Hz}$.

In the case of oscillatory eye movements, it is possible to refer to the Shannon-Nyquist statement, which indicates that the sampling frequency should be at least twice higher than the frequency of the recorded motion. Considering the studies of continuous movements, it is necessary to define purpose and intended measurement accuracy. But generally it could be assumed, that faster systems are needed to measure faster eye movements.

The most popular parameters measured in eyetracking studies are fixations and saccades. Fixation is a case, in which the eye focuses on a specific area. The subject perceives in a precise way only a small part of its field of view (about 1.5 degree). There is assumed that measurement of fixation includes also the measurement of prioritizing object in the field of vision and its cognitive processing, or more generally defining a transfer of attention to this object.

During the fixation, there are three types of slow-motion movements, so-called "interfixation movements", which could be observed:

- tremors with a frequency of about $90 \mathrm{~Hz}$,

- drift, taking the eye from the centre of fixation,

- microsaccades causing the back of the eye to its target position. 
Rapid displacement of the eye from one fixation point to another is called saccade. This is one of the fastest movements performed by the human body, lasting 30 to $80 \mathrm{~ms}$. We can say that during the saccade a lack of focus on any environment point makes that a human sees only the location of the initial and final points designated by saccade. Eye movement during a saccade rarely takes the shortest way - it can take several different shapes and curves. A large part of saccades does not stop immediately at the point of interest; eyeball oscillates briefly around or slowly moves to the point of fixation. These transitional movements between saccades and fixations are called glissades.

In the case of slow following with gaze by a moving object (such as a bird flying in the sky) a slower movement is made, which is called smooth pursuit. Another area of the brain than in the case of saccades is responsible for this movement.

The following table characterizes the most common types of eye movements, with mean values of their measurable parameters.

Tab. 1. Comparison of eye movement events parameters [4]

\begin{tabular}{|c|c|c|c|}
\hline Type & Duration (ms) & Amplitude (o) & Velocity (o/s) \\
\hline Fixation & $200-300$ & - & - \\
\hline Saccade & $30-80$ & $4-20$ & $30-500$ \\
\hline Glissade & $10-40$ & $0.5-2$ & $20-140$ \\
\hline Smooth pursuit & - & - & $10-30$ \\
\hline Mikrosaccade & $10-30$ & $10-40^{\prime}$ & $15-100^{\prime} / \mathrm{s}$ \\
\hline Tremor & - & $<1^{\prime}$ & $20^{\prime} / \mathrm{s}$ (in top) \\
\hline Drift & $200-1000$ & $1-60^{\prime}$ & $6-25^{\prime} / \mathrm{s}$ \\
\hline Square wave jerk & 200 & $0.5-1.5$ & - \\
\hline
\end{tabular}

Available eyetracking technology allows performing measurements of all parameters of the eye movement described above. It should be noted that these movements, carried out by appropriate muscles, are in fact connected with the movements of head. During a saccade in any direction, the head moves with the eyes at the same time or a moment after their move. These manoeuvres are also measurable, but very few eyetracking devices allow their measurement.

The most important feature of eyetracking devices is the sampling frequency (measured in $\mathrm{Hz}$ ). The slowest systems - already going out of use - are characterized by sampling frequency between 25 and $30 \mathrm{~Hz}$. The fastest systems allow achieving frequencies above $1000 \mathrm{~Hz}$. There are also systems that are able to achieve even $2000 \mathrm{~Hz}$, but these are only static systems, requiring fixation of the head of the subject. The fastest mobile systems that are put on the head reach a frequency of $120 \mathrm{~Hz}$, which seems entirely adequate for considered RSA studies.

Due to the studies performed in traffic, the most significant eyetracking event that can be examined is the fixation. Fixation in fact is not a movement, but an event in which the eye remains in a temporary immobility (related to the observed point), so all eyetracking systems are able to investigate this action. The saccade seems to be another important event, and because of the driving situations dynamics it also should be measured - at least approximately. Saccade is associated with moving visual perception between points of fixation. Other eye movements are not very meaningful for the driver (for example they are especially important due to the processes of reading), so it can be stated that mobile systems mounted near the subject's eye are sufficient for traffic studies.

Important factors related to the research are also the precision and the accuracy of the measurements performed by devices. Accuracy is defined as the difference between the actual gaze position, and the recorded gaze position. Precision is defined as the ability for the eye-tracker to reproduce the measurement reliably [4]. For obvious reasons, it is important for the device to be both precise and accurate. Additionally, also environmental elements and measurement method 
affects these factors, so they should be taken into account while preparing tests.

The best results in this area are achieved by the static systems that have fixed subject's head. In laboratories, the tests are carried meeting relevant requirements for illumination, and the images are at a constant distance from the subject (usually displayed on a monitor screen). The lower quality of data is obtained from mobile systems, which used in "natural" environments are exposed to heterogeneous lighting (what also can change very quickly) and the observed objects are in very different distances from the subject (which causes additional "parallax errors"). "The worst" indicators are achieved by using remote systems, where additional errors are caused by movements of the tested person's head. The quality of data using this type of system in the road traffic (where the driver dynamically reacts to the situation, i.e. looks around at the intersection) may be insufficient for precise measurements of vision.

Another important factor about the precision is spatial resolution that affects the smallest eye movements such as tremors or microsaccades. It is measurable as RMS - average square root of the angular distance between the measured values of successive samples. Simple eye-trackers are characterized by the RMS value of about $1^{\circ}$, while the most advanced equipment could reach even $0.01^{\circ}$. This value indicates that the ratio of the average sample in comparison to sample distorted by eyetracker's disruption is only $0.0001^{\circ}$, significantly less than the amplitude of the smallest detectable eye movement, and even below the level of eyemotor disruption. In fact, there is a requirement for microsaccades researches that RMS value should be below 0.03 , while for the exact calculation of the fixation's duration and measurement of saccades -0.05 .

To sum up, the differences between types of systems, their functional characteristics and functional parameters performance show that due to research in road traffics the best solution are mobile (installed on the head) and remote systems. Due to their functional characteristics, the impact on natural process of driving, and achieved performance measurements - especially the precision and accuracy - it is important to choose the right solution individually depending on the characteristics of the experiment and the required accuracy of measurements

\section{Eye-tracking in road traffic studies}

When considering the possibility of using eyetracking devices in studies related to road traffic, it is necessary to refer to both the theoretical and practical aspects of their use. Analysis made in the Motor Transport Institute indicates a high potential for such application of eyetracking devices from both of those perspectives.

First of all, eyetracking devices are supported by numerous different theories of the cognitive science concerning the relationship between visual stimuli and mental functions of human. This relationship is two-sided, because human creates a kind of saliency map, based on the accession processes of the physical properties of the stimulus, and simultaneously creates priories by assigning them to specific significances (top-down processes). The correct perception of the road environment by the driver has a significant influence on his behaviour on the road. Additional argument is brought by a research carried by Sivak (1996), which shows extremely high importance of visual perception in road traffic - visual stimuli in $83 \%$ affects driver behaviour on the road. Additionally the study of C. Ho and C. Spence [2] shows that the reactions of driver are fastest, if they are a result of visual stimuli or eyesight associated multisensory stimulating.

These studies show that the most significant stimulus modality for the driver is associated with the perception of the environment through the organ of vision, so in this area the actions to improve road safety should be concentrated - what forces the use of reliable tools to study this aspect.

Taking into account the practical aspect of using eyetracking devices in research of road safety analysis, the greatest potential for their application can be identified on the basis of studies that have been already made. Below there are listed briefly selected and characterized studies using eyetracking devices, which shows that their use in studies related to road traffic is reasonable.

First among all worth mentioning is research "Pilot Study of Instrumentation to Collect 
Behavioural Data to Identify On-Road Rider Behaviour" of NHTSA, performed in 2011 [12] in which eye-tracking devices were examined in terms of possibility to study gaze data of motorcycle riders. The results of tests were described as positive, and despite the fact that the research was provided to identify possibilities of use eye-trackers for motorcyclist studies, it shows that eyetrackers could be successfully used even on a motorcycle during the real-world traffic drive. In that context, research using mobile eye-trackers at drivers in passenger car (or truck/bus) seems to remain even more feasible and reasonable. The results of these studies indicate basis to prepare real-traffic eye-tracking experiments.

Another research worth mentioning was performed by University of Calgary and prepared at 2005 under contract with the Transportation Development Centre of Transport Canada. During the research there were made measurements, which were used to evaluate driver attention of regular road signs in comparison to attention when using a dedicated in-vehicle ITS system, showing the signs at the special display (HUD). [11]

During research, a driving simulator and head-mounted Applied Sciences Laboratory (ASL) 501 system were used. The driving simulation dependent variables measured included initial velocity at yellow light onset, velocity at stop-line, velocity at intersection exit, perception response time (PRT), perception time, response time, stopping accuracy, rate of deceleration, and intersection clearance. The eye movement variables included horizontal and vertical gaze variability, fixation percent to the HUD, and number of fixations to the HUD. The independent variables were time to stop-line.

Other research, which is very important due to the study subject, was prepared in 2010 by the Department of Traffic and Engineering Psychology of TU Braunschweig. During the research there was used also a driving simulator and eye-tracking system Dikablis. At experiment, the driver had the task to drive through the T-intersection, turning right, while different conditions of traffic occurred. [9]

Tests were designed in matrix $2 \times 2$ where from one side there have been changed number of Areas of Interest (AI) - there were one or two AI's: other vehicles from the left, and pedestrians at the right - and traffic density - two different densities of traffic from the left at the intersection: with about 70 or about 110 meters between successive vehicle.

Research was used to investigate features of traffic and road design, which could correspond to driver behaviour in the intersection. Measurements were made regarding driver behaviour and vehicle reactions, speed while approaching as well as turning right at the four intersection types was computed. During result, analysis authors have used the SEEV (Salience, Effort, Expectancy, Value) model of allocation of visual attention in comparison of traffic situations in which drivers were tested. Additionally, the drivers' waiting times at the intersections and time gaps when the drivers turned right were examined.

Tests were made on 40 drivers (26 male and 14 female) aged from 19 to 55 years, and mean annual mileage experience between 9000 and $12000 \mathrm{~km}$.

As a result of studies there was confirmed, according to SEEV model, that the higher event rate of task-relevant information (higher traffic density) grabs the driver's attention mainly and frequently to that source of information. Lower event rate conditions result in lower "interest" of driver at this area. Also, there was observed that second AI is likely to change gaze direction of driver significantly only when first (main) source of information (vehicles from the left side) would have lower event rate.

As indicated by the last one example especially, eye-trackers are successfully used in road traffic studies both covering car simulator and real-world traffic based types of research. The range of received data could be very wide, depending on the needs of experiment and first of all study subject.

\section{Legal aspects of Road Safety Audits in Poland}

The main argument for the use of road safety audits is their relatively low cost, both in relation to the high social and economic benefits is brought by their application, as well as to the overall 
cost of the road. According to the evaluation of the National Road Safety Council, the average cost of a road accident in Poland reached a level of about 1 million PLN. According to the EP strategy concerning the improvement of road safety through the use of regular road safety audits, these actions should enable a significant reduction in the number of fatalities as well as injuries and reduction of social and economic costs by identifying road sections with a high potential for possible improvement of the road safety.

Currently, the document used in GDDKiA structures about using evaluation and audits of road safety is directive of the General Director No. 42 of 3 September 2009. This document describes the procedures for carrying out evaluation and audits, required documents and their ranges. Annexes to this document indicates more detailed components and way of carrying evaluations and audits of road safety analysis, but they do not introduce a unitary methodology and do not show the required "reliability" of the analysis. Some city councils - in preparation for the implementation of the Directive - also introduce suitable directives for the management of safety in road transport, but they are usually the equivalent of the directive No. 42.

In annex 1 part. 2 to the General Director directive, there have been described detailed phases, documentation and range of audit, procedures for the preparation of audit and the content of documentation of the road safety audit process. The document also includes a list of helpful control questions for auditors as well as the list of common errors and defects that are found in the implementation of plans and road projects.

EU law, especially the Directive 2008/96/EC does not impose specific standards and methodology for evaluations and audits of road safety, leaving some flexibility in scope of their use in different countries. The annexes to the Directive indicate a list of components that should be taken into account while carrying out evaluation and audits and that should be included in their documentation. EP through unimposing specific solutions, left some flexibility in the application of audits "best practices", and at the same time more not normalized - and therefore not banned using of additional devices and tools to support the work of the auditor.

Similarly, amendment to Polish law - the Public Roads Act of 21st March 1985 (Dz.U. z 2005 nr 108 poz. 908), which introduced the road safety evaluation and road safety audits to the law regulation, does not prohibit possibility of using tools that could support the auditor's work. Despite greater requirements clarification for the evaluation and audit - in comparison to the Directive - it still leaves some flexibility in choosing particular research methods.

Reliability and quality of evaluation and road safety audits is highly dependent on the knowledge and skills of the auditor. This fact is supported by a strong emphasis set on the training process and the relatively high requirements that must be complied by an auditor.

\section{Summary}

An auditor carrying out the road safety audit should indicate both: errors arising from wrong application of legal rules concerning design and construction of roads, and as an expert, should assess the possibilities of improving safety by changing certain elements of infrastructure, or even the entire route of the road. This range of obligations indicates high responsibility of the auditor.

Complementary to the auditor's knowledge may be the use of eyetracking devices described in the article. Recent studies shows the validity of using eyetracking techniques in analyzing drivers' behaviour, both in devices used in the vehicle, as well as in relation to roads environment. It is difficult to show the clear contraindications to the use of eyetracking devices in road safety analysis, due to both the safety aspects, as well as economic reasons. The scope of information that may be obtained by using them - and what is most important data with a practical and no longer purely theoretical nature - could significantly affect the quality of research, and thus increase the effectiveness of audits.

Eyetracking systems, currently offered on the market, reach very different parameters of measurement, but due to their usefulness of the research carried out in road traffic, remote and 
mobile systems based on non-contact optical technology can be distinguished as those which meet functional requirements for research in the natural environment (during driving in normal traffic).

The use of these devices, especially in research aimed at improving road safety, requires - not developed yet - strict procedures of their usage and research methodology that could ensure the reliability of obtained results. The development of such a "unified" methodology for research in traffic will be an important stage in the development of eyetracking research in study about elements of traffic system.

To sum up the considerations presented in this article, authors conclude that the use of eyetracking devices in road safety analysis seems to be reasonable and could be another step towards improving road safety in the EU.

\section{References}

[1] Tarnowski, A., Pamięć robocza i mechanizmy kontroli ruchów oka, 300 milisekund z życia człowieka, Wydawnictwo Instytutu Psychologii Polskiej Akademii Nauk, 2009.

[2] Ho, C., Spence, C., Multisensory Driver, Human Factors in Road and Rail Transport, pp. 1-35, Ashgate 2008.

[3] Castro, C., Visual Demands and Driving, in Castro C. (editor) Visual and Cognitive Performance in Driving, pp. 1-31, Taylor \& Francis Group CRC Press 2009.

[4] Holmqvist, K. et al., Eye-Tracking, Oxford University Press, pp. 110-143, New York 2011.

[5] Bonaiuto, J., Itti, L., The Use of Attention and Spatial Information for Rapid Facial Recognition in Video, Image and Vision Computing, Vol. 24, Is. 6, pp. 557-563, 2006.

[6] Highway Capacity Manual, (HCM2010), Transportation Research Board 2010.

[7] Underwood, G., Crundall, D., Driving Simulator Validation with Hazard Perception, Transportation Research Part F [in press].

[8] Liu, B., Sun, L., Rong, J., Driver's Visual Cognition Behaviours of Traffic Signs Based on Eye Movement Parameters, Journal of Transportation Research Engineering and Information Technology, Vol. 11, Beijing 2011.

[9] Werneke, J., Vollrath, M., What Does the Driver Look at? The Influence of Intersection Characteristics on Attention Allocation and Driving Behaviour, Accident Analysis and Prevention, Germany 2011.

[10] Di Stasi, L. L., et al., Behavioural and Eye-Movement Measures to Track Improvements in Driving Skills of Vulnerable Road Users: First-Time Motorcycle Riders, Transportation Research Part F, 2011.

[11] Caird, J. K., Chisholm, S. L., Lockhart, J., Do In-Vehicle Advanced Signs Enhance Older and Younger Drivers' Intersection Performance? Driving Simulation and Eye Movement Results, International Journal Human-Computer Studies Vol. 66, 2008.

[12] Shane, B., et al., Pilot Study of Instrumentation to Collect Behavioural Data to Identify OnRoad Rider Behaviour, NHTSA, USA 2011. 